\title{
Annotations
}

\section{When not to do a lumbar puncture}

It may seem a strange principle to enunciate as the very first requirement in a Hospital that it should do the sick no harm.

FLORENCE NIGHTINGALE Notes on Hospitals, 1859

There are three reasons for not performing any clinical investigation:

(1) its inconvenience, discomfort, or expense is disproportionate to its clinical value; (2) it is unlikely to produce clinically useful information; or (3) it is unjustifiably dangerous. Any one of these considerations may apply to lumbar puncture. I do not, for instance, authorise a lumbar puncture for a toddler who seems perfectly well after a febrile convulsion, though I am eager to do so if there is any clinical doubt or in a very young child. There are many occasions (such as in most cases of epilepsy) when lumbar puncture is unlikely to give information that is sufficiently important to justify the assault on the child. Such a decision is easy, however, compared with deciding whether a lumbar puncture is or is not too dangerous. When the child is not critically ill there is little problem. Any symptoms or signs which could suggest an intracranial lesion or raised intracranial pressure will be investigated by computed tomography before lumbar puncture is considered, for example, suspicious headache or vomiting, ataxia, or hemiparesis. Symptoms of Guillain-Barré syndrome or subacute sclerosing panencephalitis, however, might justify lumbar puncture without tomography, but the only urgent indication for lumbar puncture is the suspicion of bacterial meningitis.

\section{Lumbar puncture in meningitis}

Until recently the need for lumbar puncture in diagnosing meningitis was accepted almost without question. ${ }^{1}$ In September 1985 the British Medical Journal published a letter from $\mathrm{Dr} J \mathrm{R}$ Harper describing a child with meningococcal meningitis whose condition deteriorated rapidly two hours after lumbar puncture and who subsequently died. $\mathrm{He}$ asked whether it was permissible to avoid lumbar puncture if the clinical diagnosis of meningitis seemed clear. The editor asked four experts to comment; two (a paediatrician and a physician) accepted that if there was a clear clinical diagnosis treatment should be given and lumbar puncture avoided, and two (a paediatrician and a microbiologist) thought that diagnostic lumbar puncture should be routine. ${ }^{2}$

In subsequent correspondence ${ }^{3-5}$ most writers considered that treatment of meningitis without lumbar puncture was indicated, at least in some circumstances, and there was guarded support for this approach in Australia. ${ }^{6}$ A recent comprehensive review from the USA advocated withholding or delaying lumbar puncture when there were signs of raised intracranial pressure, when there was serious cardiorespiratory disease, or when the skin over the puncture site was infected. ${ }^{7}$ Some paediatricians have reported the successful treatment of meningitis without lumbar puncture. ${ }^{8}$

Why do a lumbar puncture? Reasons given for retaining routine lumbar punctures are: to confirm the diagnosis, to identify the organism, to test for antibiotic sensitivities, and to rationalise the treatment of contacts in the case of meningococcal or haemophilus meningitis.

Paediatricians differ about the reliability of the clinical diagnosis of meningitis. Blood culture identified the organism in over $80 \%$ of the cases due to Streptococcus pneumoniae or Haemophilus influen$z a e$ at the Birmingham Children's Hospital, but failed to do so in over half of the cases due to Neisseria meningitidis. ${ }^{9}$ Organisms may be identified from smears from skin lesions, but the reliability of this method has not been determined.

What important diagnosis might be missed by not doing a lumbar puncture? Intracranial tumour and acute encephalopathy, such as Reyeis syndrome, have been mentioned as differential diagnoses, ${ }^{3}$ but for neither is there an indication for lumbar puncture. If herpes encephalitis is suspected acyclovir should be given, antibody titres to herpes virus measured, and electroencephalography and computed tomography carried out. ${ }^{10}$ Spontaneous subarachnoid haemorrhage may be difficult to diagnose, but the acute onset, fundal abnormalities, focal neurological signs, and absence of signs of infection would usually point to the correct diagno- 
sis without the need for early diagnostic lumbar puncture. ${ }^{1}$ In a young child intracranial injury may be a possibility, but again lumbar puncture is not indicated. Intracranial abscess must always be considered, whether or not the cerebrospinal fluid is infected and tomography or radionucleotide scan carried out. Clear fluid might intensify a search for metabolic disease, but the blood glucose concentration should always be checked; serum concentrations of calcium, magnesium, ammonia, and amino acids, and toxicological analysis of urine or blood may also be indicated.

Not doing a lumbar puncture means that the clinician will need to maintain a greater degree of diagnostic alertness over a longer period. Treatment with chloramphenicol will usually be adequate in meningitis caused by one of the usual three organisms, ${ }^{1 P}$ so identifying the organism in the cerebrospinal fluid is not absolutely essential. Resistance to chloramphenicol is rare, and a change of antibiotic will be dictated by lack of clinical response rather than by in vitro sensitivities. Prophylaxis for contacts is often advised for cases of meningococcal or haemophilus meningitis. Most haemophilus infections and about half of those due to meningococcus will be identified from blood cultures. ${ }^{9}$ If not, cultures of nose and throat swabs may indicate which contacts should be given rifampicin. Abandoning routine lumbar puncture would result in a loss of important epidemiological data, especially about meningococcal disease, but such a consideration will not weigh heavily with the clinician faced with an individual sick child.

The reasons for not doing a lumbar puncture. The main reason for not doing a lumbar puncture for suspected meningitis is the fear of transtentorial or transforaminal herniation or coning. We all accept that this can occur and may prove fatal, but can we estimate the risk and can we define the circumstances in which the risks outweigh the benefits?

Coning results from raised intracranial pressure caused by either cerebral oedema or acute hydrocephalus and may occur even if a lumbar puncture has not been done. Because for many years lumbar puncture was mandatory in meningitis, however, most cases of coning have followed lumbar puncture, so it is not possible to know how many would have happened anyway. Signs may develop soon after lumbar puncture and it is likely that the lumbar puncture can only make the coning worse. While it may be wise to withdraw only a small amount of fluid at diagnostic lumbar puncture, the amount is not critical because however carefully it is done fluid may continue to leak through the punctured meninges after the procedure.
Williams et al in 1964 described six children with bacterial meningitis and signs of brain stem compression, two of whom showed a dramatic improvement after treatment with hypertonic urea given intravenously. ${ }^{12}$ They gave no estimate of the proportion of their children with meningitis who developed coning.

Horwitz et al examined the records of 302 children admitted to hospital in Cleveland, Ohio, with bacterial meningitis. Coning was suspected clinically in 27. Ten children died, three of whom had had signs of coning. Many of those with coning diagnosed clinically improved after treatment with mannitol and dexamethasone, but $27 \%$ of the survivors had severe neurological damage. The authors could not identify any features on presentation that would have predicted the likelihood of coning. ${ }^{13}$

Slack found evidence of coning in six of the 90 deaths from meningococcal infection in all age groups in England and Wales in 1978. ${ }^{14}$ One of the patients had not had a lumbar puncture, but in three meningococcal meningitis had been diagnosed before lumbar puncture. In Glasgow at least five of 11 deaths in 248 children with bacterial meningitis were thought to have been associated with brain swelling and coning, ${ }^{15}$ and in a recent six month period two children with coning after lumbar puncture for meningitis were referred to the intensive care unit of the Hospital for Sick Children, Great Ormond Street. ${ }^{16}$

There is evidence, therefore, that coning may contribute to the outcome in an appreciable number of deaths from bacterial meningitis-perhaps $30 \%$ or more. It is difficult to predict which children are at particular risk, although signs suggestive of raised intracranial pressure, such as impairment of consciousness, recurrent vomiting, or focal neurological signs, would raise suspicions. Fundal signs of raised pressure alone are not adequate, but further evidence of incipient coning would make lumbar puncture a foolhardy procedure. Such signs include deterioration in the conscious level, decerebrate or decorticate rigidity, tonic seizures, unilateral or bilateral fixed dilated pupils, loss or paresis of ocular movements, hemiparesis, apnoea or irregular respiration, and extensor plantar responses. Measures to reduce intracranial pressure should be taken immediately coning is suspected.

\section{A personal approach}

In the past it was my policy to insist on lumbar puncture for all children suspected of having meningitis. I shall continue to do so for a very young child who has had a febrile convulsion or for one 
who does not rapidly recover and for a child with an acute feverish illness when the diagnosis is uncertain but meningitis is a possibility.

I shall consider treatment without lumbar puncture when the diagnosis of meningitis seems clear and the child is seriously ill, has a typical purpuric rash, has fundoscopic evidence of raised intracranial pressure, has impaired consciousness, has other signs of incipient coning, or has been ill for several days.

The debate will continue, but any decision about lumbar puncture must be made by an experienced doctor.

\section{References}

1 American College of Physicians. Health and Public Policy Committee. The diagnostic spinal tap. Ann Intern Med 1986;104:880-5

2 Harper JR, Lorber J, Hillas-Smith G, Bower BD. Eykyn SJ. Timing of lumbar puncture in severe childhood meningitis. $\mathrm{Br}$ Med J 1985;291:651-2.

${ }^{3}$ Spender Q, Thomson APJ, Jaffe IP, et al. Timing of lumbar puncture in severe childhood meningitis. $\mathrm{Br}$ Med $\mathrm{J}$ 1985; 291:898-9.

${ }^{4}$ Stephenson JBP. George RH. McWilliam R. Timing of lumbar puncture in severe childhood meningitis. $\mathrm{Br}$ Med $\mathrm{J}$ 1985; 291:1123-4.
5 Metcalf PJ. Timing of lumbar puncture in severe childhood meningitis. Br Med J 1985:291:1355.

${ }^{6}$ Silberstein P. Lumbar puncture in meningitis. Med J Aust 1986:144:110-1.

${ }^{7}$ Klein JO, Feigin RD. McCracken GH. Report of the task force on diagnosis and management of meningitis. Pediatrics 1986: 78(suppl): $959-82$

* Saced M. Wyatt GP. Dangers of lumbar puncture. Br Med J 1986;292:1740.

${ }^{9}$ George RH. Timing of lumbar puncture in severe childhood meningitis. Br Med $J$ 1985;291:1123.

11) Brett EM. Herpes simplex virus encephalitis in children. Br Med J 1986;293:1388-9.

11 Shann F. Barker J. Poore P. Chloramphenicol alone versus chloramphenicol plus penicillin for bacterial meningitis in children. Lancet 1985;ii:681-4.

12 Williams CPS, Swanson AG, Chapman JT. Brain swelling with acute purulent meningitis. Pediatrics 1964:34:220-7.

${ }^{13}$ Horwitz SJ, Boxerbaum B, O'Bell J. Cerebral herniation in bacterial meningitis in childhood. Ann Neurol 1980:7:524-8.

${ }^{14}$ Slack J. Coning and lumbar puncture. Lancet 1980:ii:474-5.

15 Stephenson JBP. Timing of lumbar puncture in severe childhood meningitis. Br Med J 1985:291:1123.

is Dezateux C. Dinwiddie R. Matthew DJ. Dangers of lumbar puncture. Br Med J 1986;292:827-8.
D P ADDY

Dudley Road Hospital, Birmingham B18 $7 \mathrm{QH}$ 
IVU and DMSA scan and found the DMSA scan more sensitive for detecting established pyelonephritis in older children. ${ }^{4}$

It is now my practice to arrange a DMSA scan before a cystogram in all children over 1 year of age. There is no doubt that a DMSA scan is less traumatic to the child (and the radiologist) than a cystogram and should therefore be placed earlier in the diagnostic sequence. An abnormal DMSA scan is an absolute indication to proceed to a cystogram as more than two thirds of the children will have reflux. If the DMSA scan is normal reflux will be a rare event and probably of little long term significance, but a careful prospective study would be required to establish this point. In the meantime, I would urge paediatricians to request DMSA scans from their radiologist colleagues who will probably be delighted to carry out these relatively non- invasive tests particularly if such a change in policy results in fewer cystograms in the toddler age group.

References

1 White RHR. Management of urinary tract infection. Arch Dis Child 1987;62:421-7.

${ }^{2}$ Haycock GB. Investigation of urinary tract infection. Arch Dis Child 1986;61:1155-8.

${ }^{3}$ Meller ST. Focal bacterial nephritis in infancy: scintigraphic appearances. Nuc Med Commun 1982;3:111.

${ }^{4}$ Merrick MV, Uttley WS, Wild SR. The detection of pyelonephritic scarring in children by radioisotope imaging. $\mathrm{Br} J$ Radiol 1980;53:544-56.

S T MELLER

Queen Mary's Hospital for Children, Carshalton, Surrey SM5 4NR

\section{Corrections}

\section{When not to do a lumbar puncture}

In the annotation entitled 'When not to do a lumbar puncture' by DP Addy (Arch Dis Child 1987;62: 873-5) we apologise that the meaning of some sentences in the published version were not clear. The original version follows.

Page 874. Second sentence of second paragraph: Treatment including chloramphenicol will usually be adequate in meningitis caused by any of the usual three organisms. ${ }^{11}$

Page 874. Final sentence of left-hand column:

Whilst it may be wise to remove only a small amount of CSF when diagnostic lumbar puncture is performed, 'careful' lumbar puncture is not the answer since CSF leak through the punctured meninges may persist after the procedure.

Page 874. Third sentence of fourth paragraph: Fundal signs of raised pressure are not to be relied upon but evidence of incipient coning would make lumbar puncture a foolhardy procedure.

\section{Page 875. Penultimate paragraph:}

I shall consider treatment without lumbar puncture: when the diagnosis of meningitis seems clear and the child is very ill, or has a typical purpuric rash, or there is fundoscopic evidence of raised pressure, or there is impairment of consciousness, or there are other signs of incipient coning, or the child has been ill for several days.

\section{Acarboxyprothrombin activity after oral prophylactic vitamin $K$}

In the paper 'Acarboxyprothrombin activity after oral prophylactic vitamin K' by von Kries, Kreppel, Becker, Tangermann, and Göbel (Arch Dis Child 1987;62:938-40) we apologise that the word 'activity' should have been published as 'concentration' throughout the paper. 